\title{
The genome sequence of the drone fly, Eristalis tenax
}

\section{(Linnaeus, 1758) [version 1; peer review: 1 approved, 1}

\section{approved with reservations]}

\author{
William Hawkes', Karl Wotton (iD), \\ University of Oxford and Wytham Woods Genome Acquisition Lab, \\ Darwin Tree of Life Barcoding collective, \\ Wellcome Sanger Institute Tree of Life programme, \\ Wellcome Sanger Institute Scientific Operations: DNA Pipelines collective, \\ Tree of Life Core Informatics collective, Darwin Tree of Life Consortium \\ ${ }^{1}$ Centre for Ecology and Conservation, University of Exeter, Penryn, UK
}

V1 First published: 10 Nov 2021, 6:307

https://doi.org/10.12688/wellcomeopenres.17357.1

Latest published: 10 Nov 2021, 6:307

https://doi.org/10.12688/wellcomeopenres.17357.1

\section{Abstract}

We present a genome assembly from an individual female Eristalis tenax (the drone fly; Arthropoda; Insecta; Diptera; Syriphidae). The genome sequence is 487 megabases in span. The majority of the assembly (96.50\%) is scaffolded into six chromosomal pseudomolecules, with the $X$ sex chromosome assembled.

Keywords

Eristalis tenax, drone fly, genome sequence, chromosomal, Diptera

This article is included in the Tree of Life gateway.

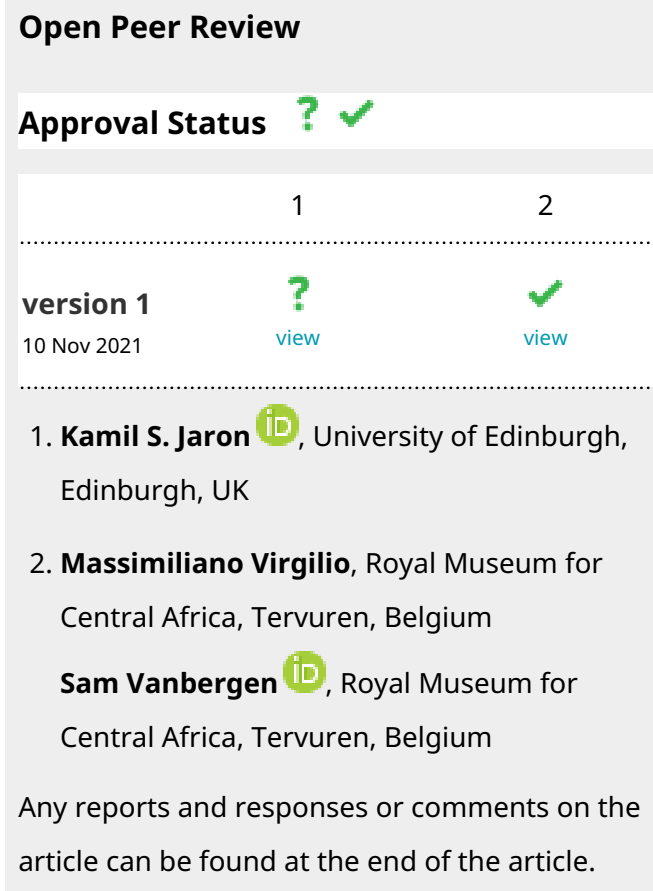

2

version 1

10 Nov 2021

view

1. Kamil S. Jaron ID, University of Edinburgh,

Edinburgh, UK

2. Massimiliano Virgilio, Royal Museum for Central Africa, Tervuren, Belgium

Sam Vanbergen iD, Royal Museum for Central Africa, Tervuren, Belgium

Any reports and responses or comments on the article can be found at the end of the article. 
Corresponding author: Darwin Tree of Life Consortium (mark.blaxter@sanger.ac.uk)

Author roles: Hawkes W: Investigation, Resources, Writing - Original Draft Preparation, Writing - Review \& Editing; Wotton K: Investigation, Supervision, Writing - Original Draft Preparation, Writing - Review \& Editing;

Competing interests: No competing interests were disclosed.

Grant information: This work was supported by Wellcome through core funding to the Wellcome Sanger Institute (206194) and the Darwin Tree of Life Discretionary Award (218328).

The funders had no role in study design, data collection and analysis, decision to publish, or preparation of the manuscript.

Copyright: (c) 2021 Hawkes W et al. This is an open access article distributed under the terms of the Creative Commons Attribution License, which permits unrestricted use, distribution, and reproduction in any medium, provided the original work is properly cited.

How to cite this article: Hawkes W, Wotton K, University of Oxford and Wytham Woods Genome Acquisition Lab et al. The genome sequence of the drone fly, Eristalis tenax (Linnaeus, 1758) [version 1; peer review: 1 approved, 1 approved with reservations] Wellcome Open Research 2021, 6:307 https://doi.org/10.12688/wellcomeopenres.17357.1

First published: 10 Nov 2021, 6:307 https://doi.org/10.12688/wellcomeopenres.17357.1 


\section{Species taxonomy}

Eukaryota; Metazoa; Ecdysozoa; Arthropoda; Hexapoda; Insecta; Pterygota; Neoptera; Endopterygota; Diptera; Brachycera; Muscomorpha; Syrphoidea; Syrphidae; Eristalinae; Eristalini; Eristalis; Eristalis tenax (Linnaeus 1758) (NCBI:txid198635).

\section{Background}

The dronefly, Eristalis tenax (Figure 1), is perhaps the most widespread of the hoverflies, with a cosmopolitan distribution. It is common in the United Kingdom and Ireland, where it can be found in the springtime following the emergence of females from overwintering in sheltered cavities in caves or buildings (Ball \& Morris, 2013). They are at their most numerous in the UK during the late summer and autumn as the local population is augmented by migratory influxes from mainland Europe. Large southward migrations have been observed during the autumn in the UK, Europe and North America (Aubert et al., 1976; Owen, 1956; Shannon, 1926). E. tenax is a fairly large hoverfly separated from others in the Eristalis genus by the presence of a
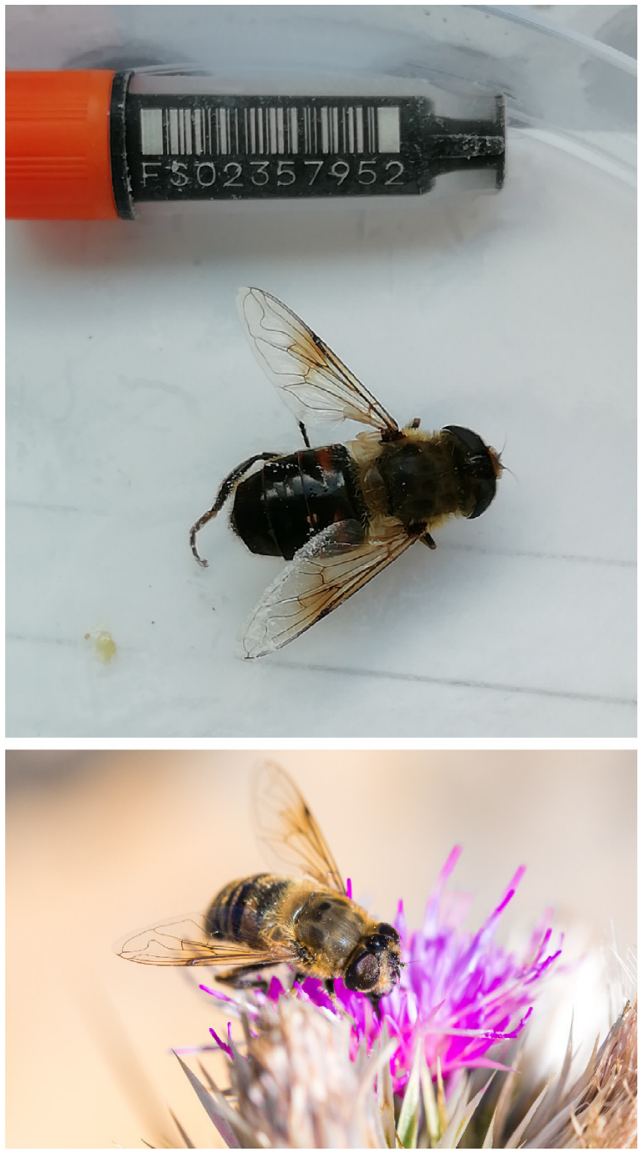

Figure 1. Top, image of the idEriTena2 specimen used for sequencing, with $43.9 \mathrm{~mm}$ FluidX sample tube for scale. Below, example image of an Eristalis tenax individual (not the sampled specimen) taken by William Hawkes. broad, black longitudinal face-stripe, longitudinal dark stripes on their eyes, and a distinctly enlarged and curved hind tibia. Like their sister species Eristalis pertinax, E. tenax is a visual, acoustic and behavioural mimic of the honeybee, Apis mellifera (Golding et al., 2001; Moore \& Hassall, 2016). Males are territorial, aggressively defending patches of flowers from conspecifics and other flying insects (Wellington \& Fitzpatrick, 1981). Their larvae, colloquially known as rat-tailed maggots, live in a wide array of organically rich pools, feeding on decaying organic matter. They therefore play an important ecological role in terms of decomposition (Hurtado et al., 2008). In addition, E. tenax is an important pollinator, visiting a wide range of crops and wild plants, while its role as a migratory pollinator may be particularly important for geographically isolated plant populations (Doyle et al., 2020; Pérez-Bañón et al., 2007; Rader et al., 2020). E. tenax can be reared and has been the subject of numerous investigations into its biology, including studies on population structure, flight, mimicry, vision and behaviour (Francuski \& Milankov, 2015; Golding et al., 2001; Lunau et al., 2018; Nicholas et al., 2018; Straw et al., 2006). This is the first production of a high quality Eristalis tenax genome, and we believe that the sequence described here, generated as part of the Darwin Tree of Life project, will further aid understanding of the biology and ecology of this hoverfly.

\section{Genome sequence report}

The genome was sequenced from a single female E. tenax collected from Wytham Great Wood, Oxfordshire, UK (latitude 51.769, longitude -1.33). A total of 36-fold coverage in Pacific Biosciences single-molecule long reads (N50 $12 \mathrm{~kb}$ ) and 60-fold coverage in 10X Genomics read clouds (from molecules with an estimated N50 of $60 \mathrm{~kb}$ ) were generated. Primary assembly contigs were scaffolded with chromosome conformation Hi-C data. Manual assembly curation corrected $71 \mathrm{missing} / \mathrm{misjoins}$ and removed 11 haplotypic duplications, reducing the assembly length by $1.92 \%$ and the scaffold number by $31.28 \%$, and increasing the scaffold $\mathrm{N} 50$ by $72.23 \%$.

The final assembly has a total length of $487 \mathrm{Mb}$ in 157 sequence scaffolds with a scaffold N50 of $77.1 \mathrm{Mb}$ (Table 1). The majority, $96.50 \%$, of the assembly sequence was assigned to 6 chromosomal-level scaffolds, representing 5 autosomes (numbered by sequence length), and the $\mathrm{X}$ sex chromosome (Figure 2-Figure 5; Table 2). The assembly has a BUSCO (Simão et al., 2015) completeness of $96.6 \%$ using the diptera_odb10 reference set. While not fully phased, the assembly deposited is of one haplotype. Contigs corresponding to the second haplotype have also been deposited.

\section{Methods}

Sample acquisition and nucleic acid extraction

A female (idEriTena2) and a male (idEriTena3) E. tenax were collected from Wytham Great Wood, Oxfordshire, UK (latitude 51.769, longitude -1.33) by Will Hawkes, University of Exeter, who also identified the sample. The samples were collected using a net, snap-frozen on dry ice and stored in a CoolRack. 
Table 1. Genome data for Eristalis tenax, idEriTena2.1.

\begin{tabular}{|c|c|}
\hline \multicolumn{2}{|l|}{ Project accession data } \\
\hline Assembly identifier & idEriTena2.1 \\
\hline Species & Eristalis tenax \\
\hline Specimen & $\begin{array}{l}\text { idEriTena2 (genome assembly, Hi-C); } \\
\text { idEriTena3 (RNA-Seq) }\end{array}$ \\
\hline NCBI taxonomy ID & 219539 \\
\hline BioProject & PRJEB43032 \\
\hline BioSample ID & SAMEA7520160 \\
\hline Isolate information & Female, head/thorax (genome assembly \\
\hline \multicolumn{2}{|l|}{ Raw data accessions } \\
\hline PacificBiosciences SEQUEL II & ERR6565938 \\
\hline 10X Genomics Illumina & ERR6054456-ERR6054459 \\
\hline Hi-C Illumina & ERR6054460 \\
\hline Illumina polyA RNA-Seq & ERR6054461 \\
\hline \multicolumn{2}{|l|}{ Genome assembly } \\
\hline Assembly accession & GCA_905231855.1 \\
\hline Accession of alternate haplotype & GCA_905231845.1 \\
\hline Span (Mb) & 487 \\
\hline Number of contigs & 290 \\
\hline Contig N50 length (Mb) & 6.65 \\
\hline Number of scaffolds & 157 \\
\hline Scaffold N50 length (Mb) & 77.07 \\
\hline Longest scaffold (Mb) & 141 \\
\hline BUSCO* genome score & $\begin{array}{l}\text { C:96.6\%[S:95.9\%,D:0.7\%],F:0.8\%, } \\
\text { M:2.6\%,n:3285 }\end{array}$ \\
\hline
\end{tabular}

*BUSCO scores based on the diptera odb10 BUSCO set using v5.1.2. C= complete [S= single copy, $D=$ duplicated], $F=$ fragmented, $M=$ missing, $n=$ number of orthologues in comparison. A full set of BUSCO scores is available at https://blobtoolkit.genomehubs. org/view/idEriTena2.1/dataset/CAJNIU01/busco.

DNA was extracted from the head/thorax of idEriTena2 at the Wellcome Sanger Institute Scientific Operations core from the whole organism using the Qiagen MagAttract HMW DNA kit, according to the manufacturer's instructions. RNA was extracted from head/thorax tissue of idEriPert3 in the Tree of Life Laboratory at the Wellcome Sanger Institute using TRIzol (Invitrogen), according to the manufacturer's instructions. RNA was then eluted in $50 \mu \mathrm{l}$ RNAse-free water and its concentration assessed using a Nanodrop spectrophotometer and Qubit Fluorometer using the Qubit RNA Broad-Range (BR) Assay kit. Analysis of the integrity of the RNA was done using Agilent RNA 6000 Pico Kit and Eukaryotic Total RNA assay.

\section{Sequencing}

Pacific Biosciences HiFi circular consensus and 10X Genomics Chromium read cloud sequencing libraries were constructed according to the manufacturers' instructions. Poly(A) RNA-Seq libraries were constructed using the NEB Ultra II RNA Library Prep kit. Sequencing was performed by the Scientific Operations core at the Wellcome Sanger Institute on Pacific Biosciences SEQUEL II (HiFi), Illumina HiSeq X (10X) and Illumina HiSeq 4000 (RNA-Seq) instruments. Hi-C data were generated from the abdomen tissue of idEriTena2 using the Arima v1 Hi-C kit and sequenced on HiSeq X. 


\section{Scaffold statistics}

\section{BUSCO diptera_odb10 (3285)}

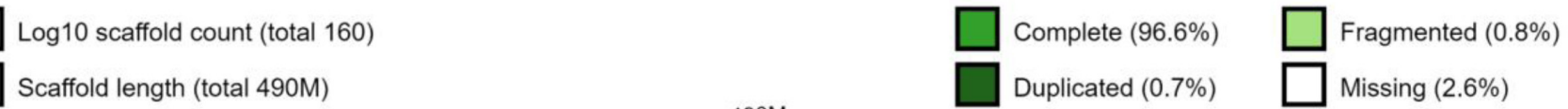

Longest scaffold (140M)

N50 length $(77 \mathrm{M})$

N90 length $(66 \mathrm{M})$

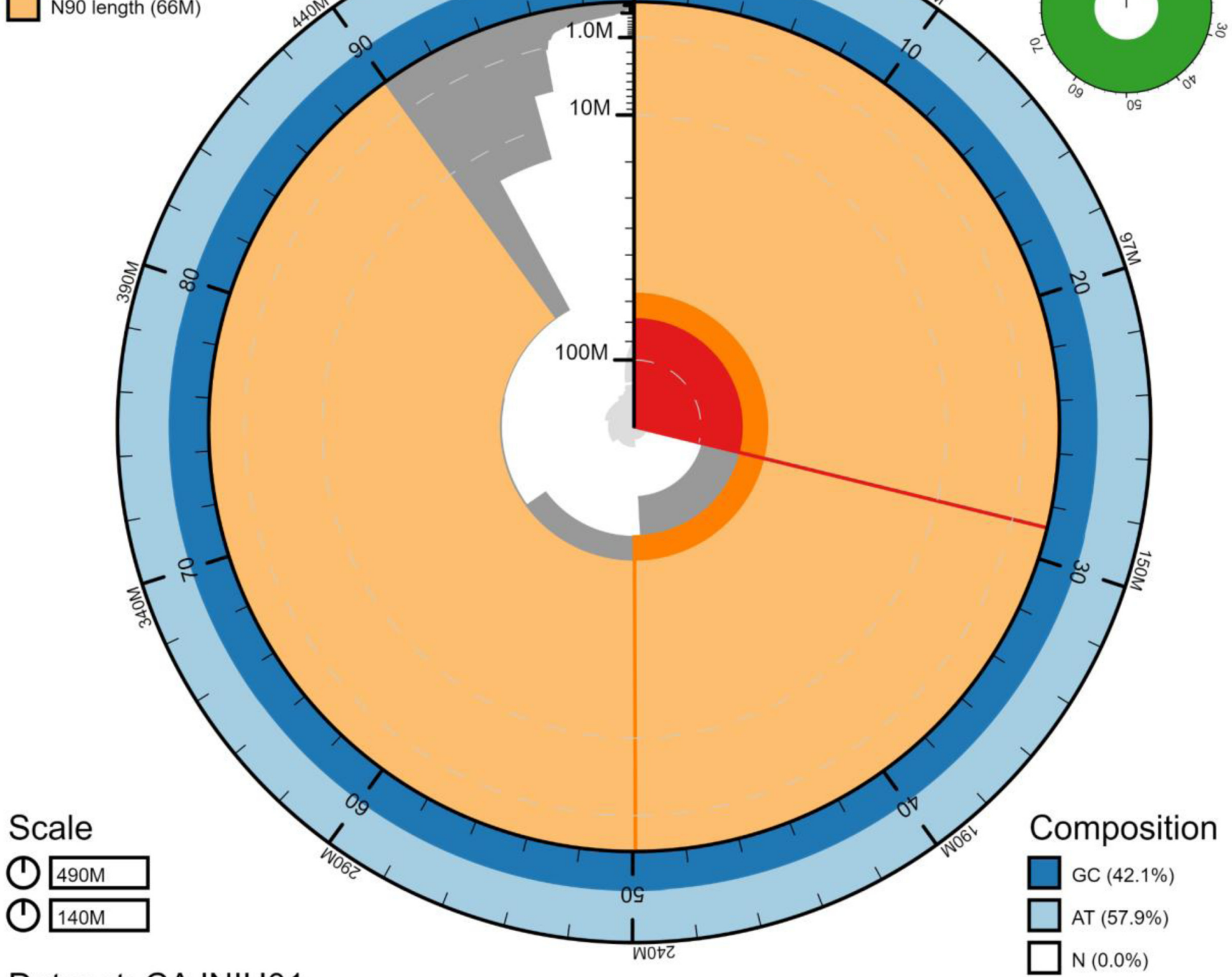

\section{Dataset: CAJNIU01}

Figure 2. Genome assembly of Eristalis tenax, idEriTena2.1: metrics. The BlobToolKit Snailplot shows N50 metrics and BUSCO gene completeness. The main plot is divided into 1,000 size-ordered bins around the circumference with each bin representing $0.1 \%$ of the $487,019,564$ bp assembly. The distribution of scaffold lengths is shown in dark grey with the plot radius scaled to the longest scaffold present in the assembly $(140,698,496$ bp, shown in red). Orange and pale-orange arcs show the N50 and N90 scaffold lengths (77,068,984 and $65,930,618 \mathrm{bp})$, respectively. The pale grey spiral shows the cumulative scaffold count on a log scale with white scale lines showing successive orders of magnitude. The blue and pale-blue area around the outside of the plot shows the distribution of GC, AT and N percentages in the same bins as the inner plot. A summary of complete, fragmented, duplicated and missing BUSCO genes in the diptera odb10 set is shown in the top right. An interactive version of this figure is available at https://blobtoolkit.genomehubs.org/view/idEriTena2.1/ dataset/CAJNIU01/snail. 


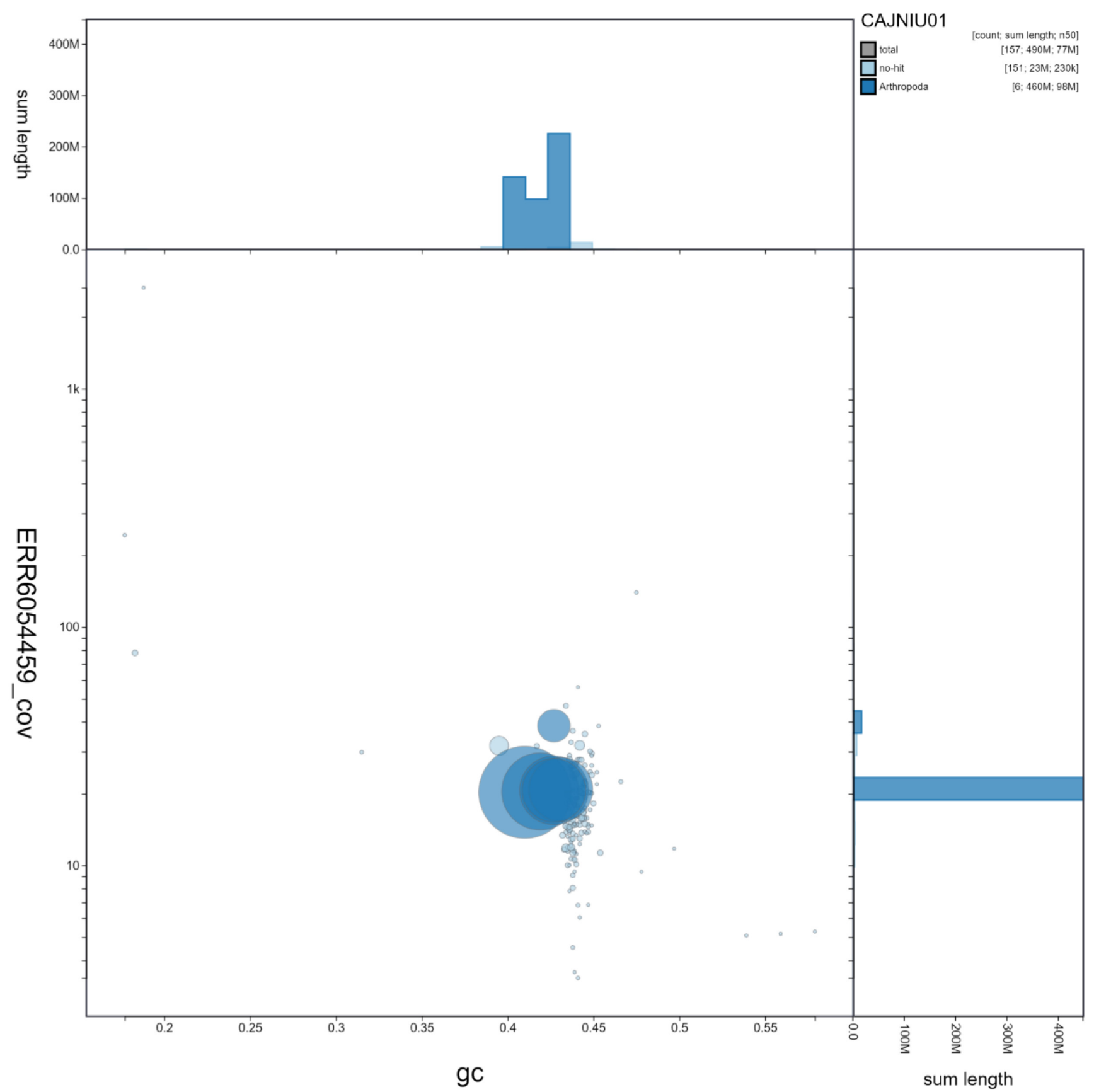

Figure 3. Genome assembly of Eristalis tenax, idEriTena2.1: GC coverage. BlobToolKit GC-coverage plot. Scaffolds are coloured by phylum. Circles are sized in proportion to scaffold length. Histograms show the distribution of scaffold length sum along each axis. An interactive version of this figure is available at https://blobtoolkit.genomehubs.org/view/idEriTena2.1/dataset/CAJNIU01/blob.

\section{Genome assembly}

Assembly was carried out with Hifiasm (Cheng et al., 2021); haplotypic duplication was identified and removed with purge_dups (Guan et al., 2020) with the -e flag. One round of polishing was performed by aligning $10 \mathrm{X}$ Genomics read data to the assembly with longranger align, calling variants with freebayes (Garrison \& Marth, 2012). The assembly was then scaffolded with Hi-C data (Rao et al., 2014) using SALSA2 (Ghurye et al., 2019). The assembly was checked for contamination and corrected using the gEVAL system (Chow et al., 2016) as described previously (Howe et al., 2021). Manual curation was performed using gEVAL, HiGlass 


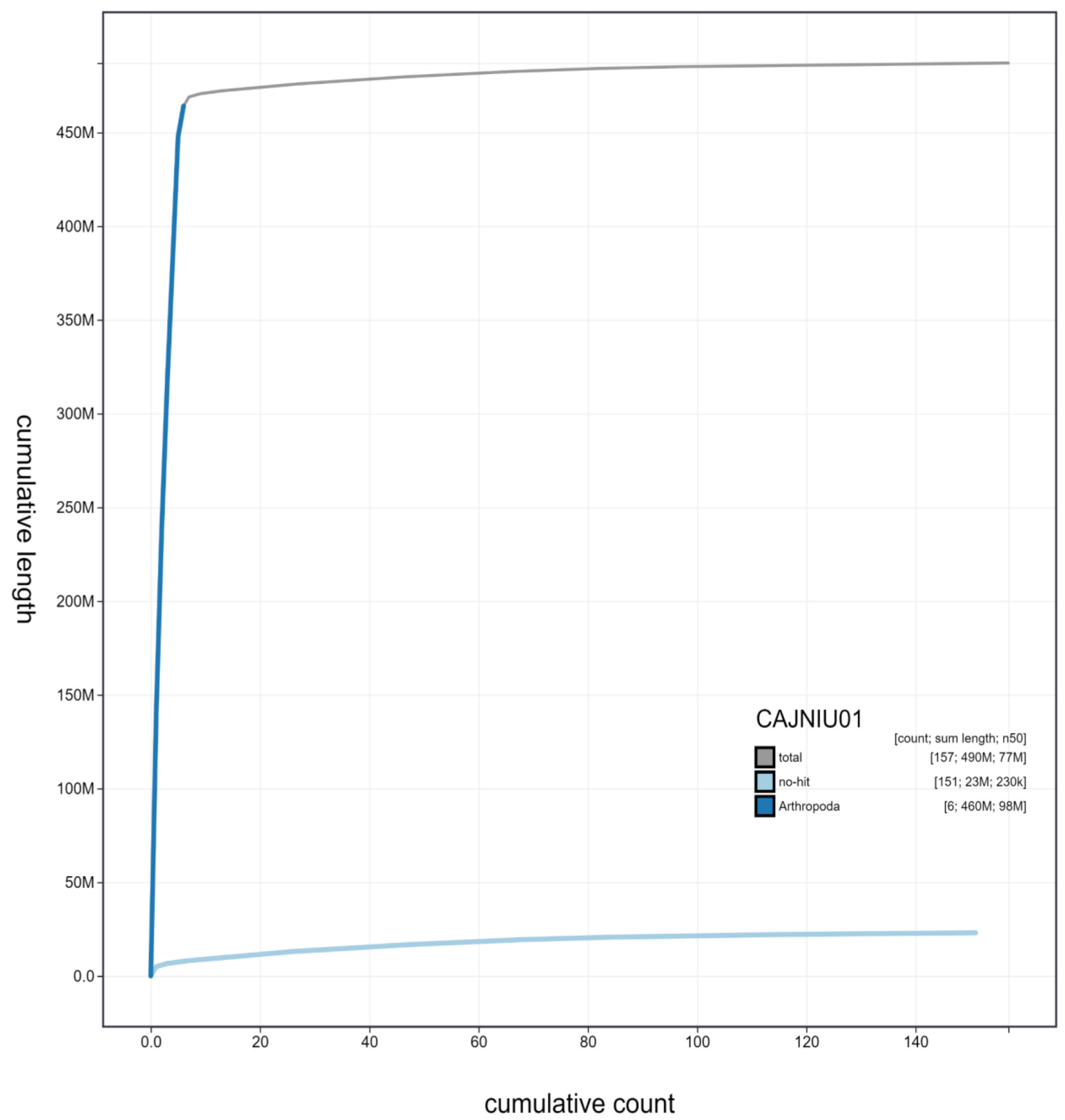

Figure 4. Genome assembly of Eristalis tenax, idEriTena2.1: cumulative sequence. BlobToolKit cumulative sequence plot. The grey line shows cumulative length for all scaffolds. Coloured lines show cumulative lengths of scaffolds assigned to each phylum using the buscogenes taxrule. An interactive version of this figure is available at https://blobtoolkit.genomehubs.org/view/idEriTena2.1/dataset/ CAJNIU01/cumulative.

(Kerpedjiev et al., 2018) and Pretext. The mitochondrial genome was assembled using MitoHiFi (Uliano-Silva et al., 2021). The genome was analysed and BUSCO scores generated within the BlobToolKit environment (Challis et al., 2020). Table 3 contains a list of all software tool versions used, where appropriate. 


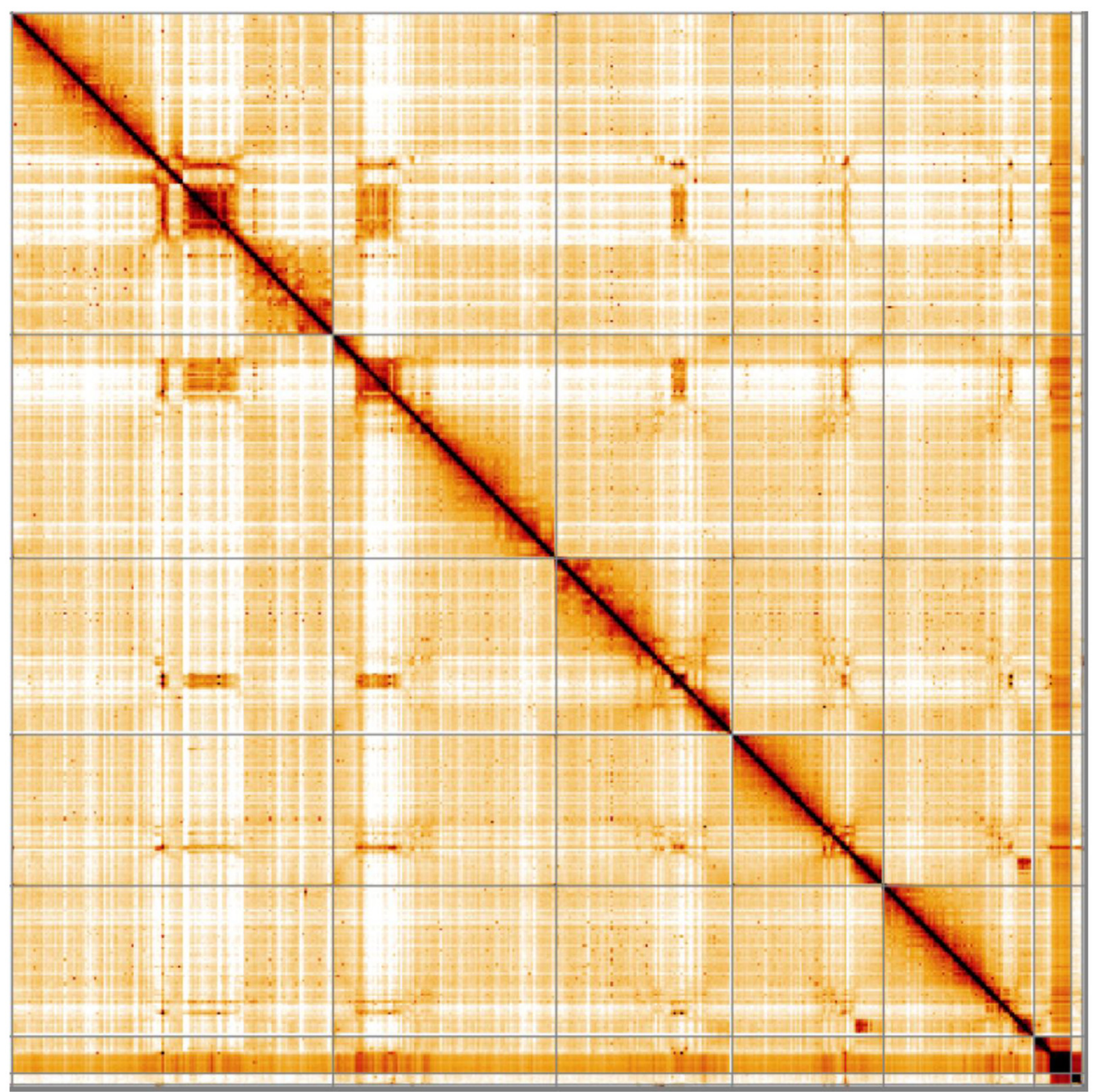

Figure 5. Genome assembly of Eristalis tenax, idEriTena2.1: Hi-C contact map. Hi-C contact map of the idEriTena2.1 assembly, visualised in HiGlass.

\begin{tabular}{|c|c|c|c|}
\hline \multicolumn{4}{|c|}{$\begin{array}{l}\text { Table 2. Chromosomal pseudomolecules in the } \\
\text { genome assembly of Eristalis tenax, idEriTena2. }\end{array}$} \\
\hline INSDC accession & Chromosome & Size (Mb) & GC\% \\
\hline HG993124.1 & 1 & 140.7 & 41 \\
\hline HG993125.1 & 2 & 97.83 & 41.9 \\
\hline HG993126.1 & 3 & 77.07 & 42.7 \\
\hline HG993127.1 & 4 & 66.31 & 43.1 \\
\hline HG993128.1 & 5 & 65.93 & 42.7 \\
\hline HG993129.1 & $X$ & 16.18 & 42.7 \\
\hline HG993130.1 & MT & 0.02 & 18.8 \\
\hline- & Unplaced & 22.99 & 42.8 \\
\hline
\end{tabular}


Table 3. Software tools used.

\begin{tabular}{|l|l|l|}
\hline Software tool & Version & Source \\
\hline Hifiasm & 0.12 & Cheng et al., 2021 \\
\hline purge_dups & 1.2 .3 & Guan et al., 2020 \\
\hline SALSA2 & 2.2 & Ghurye et al., 2019 \\
\hline Iongranger align & 2.2 .2 & $\begin{array}{l}\text { https://support.10xgenomics.com/genome-exome/ } \\
\text { software/pipelines/latest/advanced/other-pipelines }\end{array}$ \\
\hline freebayes & $1.3 .1-17-g a a 2 a c e 8$ & Garrison \& Marth, 2012 \\
\hline MitoHiFi & 1 & Uliano-Silva et al., 2021 \\
\hline gEVAL & N/A & Chow et al., 2016 \\
\hline HiGlass & 1.11 .6 & Kerpedjiev et al., 2018 \\
\hline PretextView & $0.1 . x$ & https://github.com/wtsi-hpag/PretextView \\
\hline BlobToolKit & 2.6 .2 & Challis et al., 2020 \\
\hline
\end{tabular}

\section{Data availability}

European Nucleotide Archive: Eristalis tenax (the dronefly). Accession number PRJEB43032; https://identifiers.org/ena.embl/ PRJEB43032.

The genome sequence is released openly for reuse. The E. pertinax genome sequencing initiative is part of the Darwin Tree of Life (DToL) project. All raw sequence data and the assembly have been deposited in INSDC databases. The genome will be annotated using the RNA-Seq data and presented through the Ensembl pipeline at the European Bioinformatics Institute. Raw data and assembly accession identifiers are reported in Table 1.

\section{Author information}

Members of the University of Oxford and Wytham Woods Genome Acquisition Lab are listed here: https://doi.org/10.5281/ zenodo.4789929.
Members of the Darwin Tree of Life Barcoding collective are listed here: https://doi.org/10.5281/zenodo.4893704.

Members of the Wellcome Sanger Institute Tree of Life programme are listed here: https://doi.org/10.5281/ zenodo.5377053.

Members of Wellcome Sanger Institute Scientific Operations: DNA Pipelines collective are listed here: https://doi.org/10.5281/ zenodo.4790456.

Members of the Tree of Life Core Informatics collective are listed here: https://doi.org/10.5281/zenodo.5013542.

Members of the Darwin Tree of Life Consortium are listed here: https://doi.org/10.5281/zenodo.4783559.
Aubert ], Aubert ]j, Goeldlin P: DOUZE ANS DE CAPTURES SYSTEMATIQUES DE SYRPHIDES (DIPTERES) AU COL DE BRETOLET (ALPES VALAISANNES). 1976. Reference Source

Ball S, Morris R: Britain's Hoverflies: A Field Guide (Second Edi). WILDGuides. 2013

Challis R, Richards E, Rajan J, et al.: BlobToolKit - Interactive Quality Assessment of Genome Assemblies. G3 (Bethesda). 2020; 10(4): 1361-74. PubMed Abstract | Publisher Full Text | Free Full Text

Cheng $\mathrm{H}$, Concepcion GT, Feng $X$, et al.: Haplotype-Resolved de Novo Assembly Using Phased Assembly Graphs with Hifiasm. Nat Methods. 2021; 18(2): 170-75.

PubMed Abstract | Publisher Full Text | Free Full Text

Chow W, Brugger K, Caccamo M, et al:: gEVAL - a web-based browser for evaluating genome assemblies. Bioinformatics. 2016; 32(16): 2508-10. PubMed Abstract | Publisher Full Text | Free Full Text

Doyle T, Hawkes WLS, Massy R, et al.: Pollination by Hoverflies in the Anthropocene. Proc Biol Sci. 2020; 287(1927): 20200508. PubMed Abstract | Publisher Full Text | Free Full Text

Francuski L, Milankov V: Assessing Spatial Population Structure and Heterogeneity in the Dronefly. J Zool. 2015; 297(4): 286-300. Publisher Full Text

Garrison E, Marth G: Haplotype-Based Variant Detection from Short-Read Sequencing. arXiv: 1207.3907, 2012.

Reference Source

Ghurye J, Rhie A, Walenz BP, et al.: Integrating Hi-C Links with Assembly Graphs for Chromosome-Scale Assembly. PLoS Comput Biol. 2019; 15(8): 
e1007273

PubMed Abstract | Publisher Full Text | Free Full Text

Golding YC, Ennos AR, Edmunds M: Similarity in Flight Behaviour between the Honeybee Apis Mellifera (Hymenoptera: Apidae) and Its Presumed Mimic, the Dronefly Eristalis Tenax (Diptera: Syrphidae). J Exp Biol. 2001; 204(Pt 1): 139-45.

PubMed Abstract

Guan D, McCarthy SA, Wood J, et al.: Identifying and Removing Haplotypic Duplication in Primary Genome Assemblies. Bioinformatics. 2020; 36(9): 2896-98.

PubMed Abstract | Publisher Full Text | Free Full Text

Howe K, Chow W, Collins J, et al.: Significantly Improving the Quality of Genome Assemblies through Curation. Gigascience. 2021; 10(1): giaa153. PubMed Abstract | Publisher Full Text | Free Full Text

Hurtado P, Pérez-Bañón C, Gladis T, et al.: Biology of Saprophagous Hoverflies (Diptera, Syrphidae) and Its Role in Degrading of Pig Slurry. In XXIII

International Congress of Entomology, Durban (South Africa). 2008.

Kerpedjiev P, Abdennur N, Lekschas F, et al.: HiGlass: Web-Based Visual Exploration and Analysis of Genome Interaction Maps. Genome Biol. 2018; 19(1): 125

PubMed Abstract | Publisher Full Text | Free Full Text

Lunau K, An L, Donda M, et al.: Limitations of Learning in the Proboscis Reflex of the Flower Visiting Syrphid Fly Eristalis Tenax. PLoS One. 2018; 13(3): e0194167.

PubMed Abstract | Publisher Full Text | Free Full Text

Moore CD, Hassall C: A Bee or Not a Bee: An Experimental Test of Acoustic Mimicry by Hoverflies. Behavioral Ecology: Official Journal of the International Society for Behavioral Ecology. 2016; 27(6): 1767-74.

Publisher Full Text

Nicholas S, Thyselius M, Holden M, et al.: Rearing and Long-Term Maintenance of Eristalis Tenax Hoverflies for Research Studies. J Vis Exp. 2018; (135): 57711

PubMed Abstract | Publisher Full Text | Free Full Text
Owen DF: A Migration of Insects at Spurn Point, Yorkshire. Entomologist's Monthly Magazine. 1956; 92: 43-44.

Pérez-Bañón C, Petanidou T, Ángeles Marcos-García Ma: Pollination in Small Islands by Occasional Visitors: The Case of Daucus Carota Subsp. Commutatus (Apiaceae) in the Columbretes Archipelago, Spain. Plant Ecology. 2007; 192(1): 133-51.

Publisher Full Text

Rader R, Cunningham SA, Howlett BG, et al.: Non-Bee Insects as Visitors and Pollinators of Crops: Biology, Ecology, and Management. Annu Rev Entomol. 2020; 65: 391-407.

PubMed Abstract | Publisher Full Tex

Rao SSP, Huntley MH, Durand NC, et al.: A 3D Map of the Human Genome at Kilobase Resolution Reveals Principles of Chromatin Looping. Cell. 2014; 159(7): 1665-80.

PubMed Abstract | Publisher Full Text | Free Full Text

Shannon HJ: A Preliminary Report on the Seasonal Migrations of Insects. J New York Entomol S. 1926; 34(2): 199-205.

Reference Source

Simão FA, Waterhouse RM, Ioannidis P, et al: BUSCO: Assessing Genome Assembly and Annotation Completeness with Single-Copy Orthologs. Bioinformatics. 2015: 31(19): 3210-12

PubMed Abstract | Publisher Full Tex

Straw AD, Warrant EJ, O'Carroll DC: A “bright zone” in male hoverfly (Eristalis tenax) eyes and associated faster motion detection and increased contrast sensitivity. J Exp Biol. 2006; 209(Pt 21): 4339-54.

PubMed Abstract | Publisher Full Text

Uliano-Silva M, Nunes JGF, Krasheninnikova K, et al.: marcelauliano/MitoHiFi: mitohifi v2.0. 2021.

Publisher Full Text

Wellington WG, Fitzpatrick SM: TERRITORIALITY IN THE DRONE FLY, ERISTALIS TENAX (DIPTERA: SYRPHIDAE). The Canadian Entomologist. 1981; 113(8):

695-704.

Publisher Full Text 


\section{Open Peer Review}

\section{Current Peer Review Status:}

\section{Version 1}

Reviewer Report 25 April 2022

https://doi.org/10.21956/wellcomeopenres.19191.r49837

(c) 2022 Virgilio $\mathbf{M}$ et al. This is an open access peer review report distributed under the terms of the Creative Commons Attribution License, which permits unrestricted use, distribution, and reproduction in any medium, provided the original work is properly cited.

\section{Massimiliano Virgilio}

Department of Biology, Royal Museum for Central Africa, Tervuren, Belgium

\section{Sam Vanbergen}

Royal Museum for Central Africa, Tervuren, Belgium

Technical paper presenting a first chromosome-level assembly for the cosmopolitan pollinator fly Eristalis tenax (Diptera, Syrphidae). Clearly presented wet- and dry-lab pipelines and robust methodological approach relying on well established procedures. A relevant contribution to genomic research on Syrphidae. Just for the sake of clarity, it would be nice to have additional info on the amount of DNA/RNA recovered and used in library prep.

\section{Additional input from co-reviewer S. Vanbergen (RMCA):}

There might be instances of mixed-up IDs/species names. It is mentioned "RNA was extracted from head/thorax tissue of idEriPert3", shouldn't this be IdEriTena3? In table 1 the IDs appear to be correct. Likewise, in the data availability section the "E. pertinax genome sequencing initiative" is brought up. I presume this should be the "E. tenax genome sequencing initiative" instead.

Is the rationale for creating the dataset(s) clearly described?

Yes

Are the protocols appropriate and is the work technically sound?

Yes

Are sufficient details of methods and materials provided to allow replication by others? Yes

Are the datasets clearly presented in a useable and accessible format? Yes

Competing Interests: No competing interests were disclosed. 
Reviewer Expertise: molecular ecology and systematics of Diptera, integrative -omic approaches to taxonomy

We confirm that we have read this submission and believe that we have an appropriate level of expertise to confirm that it is of an acceptable scientific standard.

Reviewer Report 14 April 2022

https://doi.org/10.21956/wellcomeopenres.19191.r49835

(C) 2022 Jaron $\mathrm{K}$. This is an open access peer review report distributed under the terms of the Creative Commons Attribution License, which permits unrestricted use, distribution, and reproduction in any medium, provided the original work is properly cited.

\section{Kamil S. Jaron}

Institute of Evolutionary Biology, University of Edinburgh, Edinburgh, UK

I very much welcome another dipteran genome for I was always interested in the remarkable diversity of sex determination in this taxon.

The only available information regarding $E$. tenax karyotype I found is http://dx.doi.org/10.13005/bbra/2672, which is suggesting $E$. tenax is has XY sex determination. Perhaps I would mention that $Y$ is missing in the assembly (given a female was sequenced). Furthermore, the same study proposes that the $X$ chromosome is actually of a similar size to other smaller autosomes, but in this assembly, it seems to be a lot shorter (16.18 Mbp compared to $60 \mathrm{Mbp}+$ ). Could you elaborate on the difference?

Related to this issue, there is no indication in the paper on how the $\mathrm{X}$ chromosome was identified in this species. How do we know which one is $X$ ?

Otherwise, It is a really interesting genome, I am very pleased to see the Chr 1 assembled in full glory in $140 \mathrm{Mbp}$ !

\section{References}

1. Khajuria M, Ayoub Bhatti A, Tripathi N: Cytogenetic Analysis of A Hoverfly Eristalis tenax (Diptera: Syrphidae). Biosciences, Biotechnology Research Asia. 2018; 15 (3): 649-653 Publisher Full Text

Is the rationale for creating the dataset(s) clearly described? Yes

Are the protocols appropriate and is the work technically sound? Partly

Are sufficient details of methods and materials provided to allow replication by others? Partly 
Are the datasets clearly presented in a useable and accessible format?

Yes

Competing Interests: No competing interests were disclosed.

Reviewer Expertise: Evolutionary genomics

I confirm that I have read this submission and believe that I have an appropriate level of expertise to confirm that it is of an acceptable scientific standard, however I have significant reservations, as outlined above. 